Open Access : ISSN : 1848-7718

http://www.pub.iapchem.org/ojs/index.php/admet/index

Review

\title{
Uric acid transporter inhibitors for gout
}

\author{
Philip K. Tan and Jeffrey N. Miner* \\ Pharmadiscovery Consulting LLC, San Diego, CA 92122 \\ *Corresponding Author: E-mail: pharmadiscoveryconsulting@gmail.com; \\ Received: April 20, 2017; Revised: May 21, 2017; Published: June 22, 2017
}

\begin{abstract}
Gout is a common inflammatory arthritis that is caused by chronically-elevated serum uric acid (sUA) levels (hyperuricemia). In humans, sUA levels are predominantly controlled by a variety of transporters that mediate the elimination of uric acid through the kidneys and intestines, a process that is altered in most gout patients. In this review, we highlight our current understanding of uric acid handling in healthy individuals and gout patients, therapies for gout that target uric acid transporters, and the mechanism of other therapies that alter sUA levels through interactions with uric acid transporters.
\end{abstract}

\section{Keywords}

Gout; hyperuricemia; uricase; URAT1; coevolution; urate transporters for urate homeostasis

\section{Overview}

\section{Gout and hyperuricemia}

Gout is a debilitating inflammatory arthritis of increasing prevalence that is caused by chronically elevated levels of serum uric acid (sUA), or hyperuricemia, defined as concentrations exceeding $6.8 \mathrm{mg} / \mathrm{dL}$ $(408 \mu \mathrm{M})$. In healthy nongouty individuals, serum urate levels are normally in the range of $3.5-7 \mathrm{mg} / \mathrm{dL}$ $(210-420 \mu \mathrm{M})$ [1]. Prolonged hyperuricemia can initiate the precipitation of the uric acid in joints and other tissues, and these deposits can trigger an acute and painful immune response known as a gout flare. Hyperuricemia is also strongly and independently associated with a number of other important disorders including hypertension, cardiovascular disease and metabolic syndrome [2]. A number of therapies for gout that lower sUA levels target transporters for uric acid, some which are also important drug transporters.

Uric acid homeostasis in humans in health and disease

Uric acid is produced mainly in the liver and gastrointestinal tract by the degradation of endogenous and dietary purines. Sources of endogenous purines include nucleotides such as ATP, and DNA and RNA that are recovered from dying cells. These purines are degraded into uric acid by xanthine oxidase in the liver. Dietary purines are absorbed in the gut by the CNT2 transporter and are quantitatively metabolized to uric acid by endogenous xanthine oxidase within intestinal enterocytes (see Figure 1b). In non-primate mammals, uric acid is converted to more highly soluble allantoin by urate oxidase (uricase). In primates including humans, however, uric acid is the end product of purine catabolism due to the progressive 
inactivation of uricase in primate evolution [3] hence primates have sUA levels that are 3-10 times higher than other mammals. The xanthine oxidase inhibitor (XOI) class of urate-lowering therapies for gout (allopurinol and febuxostat) block the enzyme xanthine oxidase that produces uric acid in the last step of purine catabolism in primates [4].

Uric acid production is balanced by elimination, which in humans occurs mainly through the kidneys ( $70 \%$ of the total eliminated uric acid) but also through the intestines (30\% of the total eliminated uric acid). With a pKa of 5.35 [5], uric acid is the negatively charged organic anion urate at physiological pH and so requires transporters for passage through cell membranes. Genetic studies show that a large number of urate transporters (GLUT9, ABCG2, NPT1/3/4, URAT1, OAT4, MRP4) are important for urate homeostasis in humans [6-8] which are expressed in the organs that eliminate uric acid. In the kidney nephrons, uric acid handling occurs in a complex process involving complete and free filtration into the glomerulus, followed by near complete $90-95 \%$ reabsorption in the proximal tubules back to the bloodstream (Figure 1a). Uric acid that is not reabsorbed in the proximal tubule (5-10\% of the glomerular-filtered uric acid) passes through the nephron and is excreted into the urine, and there is little evidence that uric acid can pass through the walls of the more distal segments of the nephron. The transporters involved in the proximal tubule handling of uric acid are shown in Figure 1a and are described in detail below.

a)

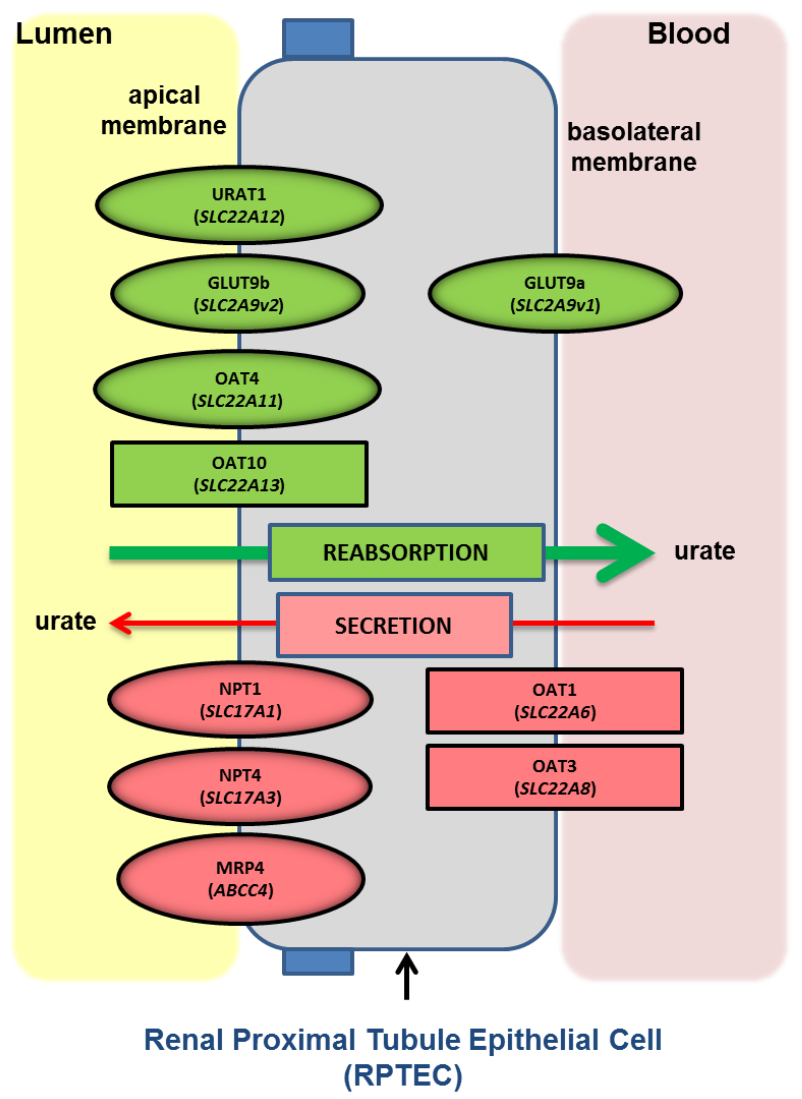

b)

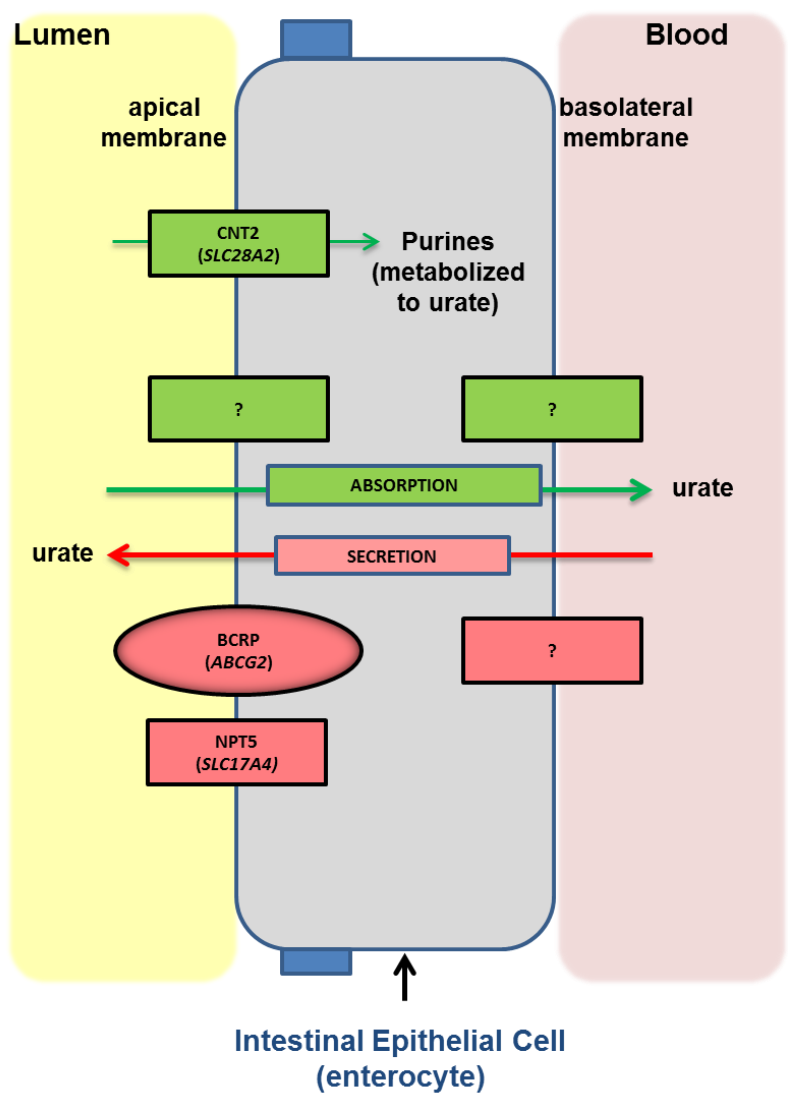

Figure 1. Organization of urate transporters in polarized epithelial cells of the kidney (a) and intestine (b). Transporters involved in reabsorption/absorption of urate are shown in green, while those involved in secretion are shown in red. Transporters in ovals are genetically-associated with sUA levels, while transporters in boxes have no genetic association with sUA levels, but transport urate in vitro. Specific urate transporters in the intestine, particularly on the basolateral membrane, are unknown. Intestinal CNT2 is involved in import of dietary purines from the intestinal lumen, which are efficiently metabolized to urate by the enterocytes. 
In the intestine, uric acid permeability from mucosal to serosal (absorption) and serosal to mucosal (secretion) has been documented, but the identities of the responsible transporters in humans are, for the most part, unknown (Figure 1b). The important drug transporter breast cancer resistance protein (BCRP, encoded by the $A B C G 2$ gene) is highly expressed on the apical surface of intestinal epithelial cells and is also an important urate transporter involved in intestinal urate secretion [9-12]. Dietary purines are imported from the lumen of intestinal enterocytes by the nucleoside transporter CNT2/SLC28A2, which are then converted to uric acid by intestinal xanthine oxidase. Inhibitors of CNT2 have been shown to reduce sUA levels in non-human primates and are therefore possible candidates for new urate-lowering therapies [13].

The chronically elevated concentrations of SUA in gout patients are caused by increased uric acid production, decreased uric acid elimination, or a combination of the two. In most gout patients, high sUA levels are due to decreased uric acid elimination in the kidneys, recently confirmed with clinical trial results [14]. We also discovered that gout patients have an altered renal urate reabsorption profile in response to $\mathrm{XOI}$ urate lowering therapies, relative to healthy individuals. These differences in the renal urate reabsorption in gout patients are likely due to differences in the activities of renal urate transporters. It is possible that transporters involved in reabsorption (colored green in Figure 1a) are more active, leading to enhanced reabsorption of urate, or that transporters involved in urate secretion (colored red in Figure 1a) are less active, or some combination of the above two scenarios [14]. It is also now known that many drugs and metabolic anomalies increase or decrease sUA levels by altering the activities of urate transporters, and this is explained in the following section.

\section{Uric acid transporters and drug interactions}

\section{URAT1}

The renal uric acid transporter type 1 (URAT1/SLC22A12) is expressed on the apical membranes of renal proximal tubule epithelial cells (RPTECS) and is required for the first step in reabsorption of uric acid from the lumen of the proximal tubule to the bloodstream (Figure 1a). Individuals carrying inactivating mutations of URAT1 have high levels of urinary uric acid excretion and low levels of sUA (renal hypouricemia) due to reduced uric acid reabsorption from the proximal tubules, showing that URAT1 is essential for efficient renal reabsorption of uric acid [15]. The uricosuric class of urate-lowering therapies (lesinurad, probenecid, and benzbromarone) for the treatment of gout lower sUA concentrations by inhibiting URAT1. Other drugs indicated primarily for other therapies (losartan, hypertension; fenofibrate, hyperlipidemia) are considered secondary uricosurics because they also inhibit URAT1 $[16,17]$.

URAT1 is a member of the organic anion transporter (OAT) subtype of solute carrier (SLC) type 22A family (SLC22A) of transporters, which typically function as organic anion exchangers. Specifically, URAT1 imports urate in exchange for export of small anionic counterions such as lactate (Figure 2). The activity of URAT1 depends on these counterions, and certain pathophysiological conditions and drugs alter sUA levels by altering the activity of URAT1. Increased concentrations of counterions can stimulate the counterion transport of URAT1, leading to increased uric acid transport of URAT1 in a process called trans-stimulation. Alcohol poisoning (lactic acidosis) and diabetic ketoacidosis elevate sUA levels due to elevated levels of the URAT1 counterions lactate and ketoacids (acetoacetate and $\beta$-hydroxybutyrate), respectively [18, 19]. Furthermore, salicylate (at low doses), niacin or nicotinate (for treatment of hyperlipidemia), and pyrazinoate (the primary metabolite of the tuberculosis therapy pyrazinamide) function as pharmacological URAT1 counterions and also elevate sUA levels through trans-stimulation of URAT1. High doses of 
salicylate, on the other hand, inhibit the urate transport activity of URAT1 (cis-inhibition) to lower sUA levels. Therefore, the paradoxical effect of salicylate dosage on sUA levels is explained through different dose-dependent interactions with URAT1.

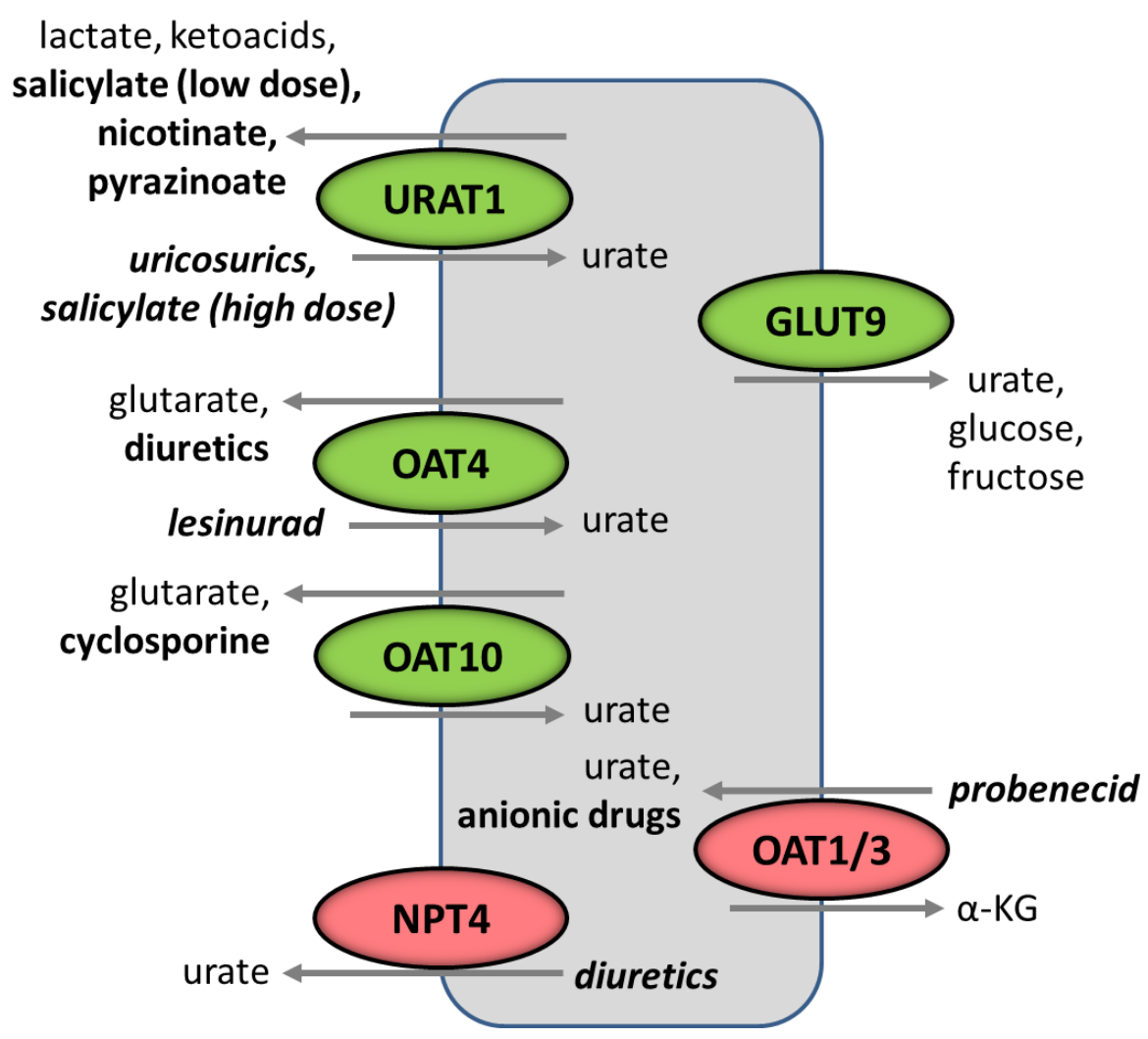

Figure 2. Effects of xenobiotics and endobiotics on urate transport in the kidney proximal tubule. For each transporter, endogenous substrates are in normal case, while drugs are in bold (nicotinate is an endogenous substrate for URAT1 but is also used as a therapy for hyperlipidemia). The proposed direction of urate transport is shown for each transporter. Drugs that stimulate transport of urate are not italicized, while drugs that inhibit urate transport are italicized. See text for more details. $\alpha-K G$, alpha-ketoglutarate.

URAT1 is classified as a tertiary active transporter, as its activity is dependent on counterion and sodium gradients generated by secondary active transporters ( $\mathrm{Na}+$ /monocarboxylate cotransporters SMCT1/2) and the primary active transporter ( $\mathrm{Na}+\mathrm{K}+$ ATPase), respectively (Figure 3). URAT1 and SMCT1/2 also interact with PDZK1, a protein involved in the localization of apical membrane proteins in kidney and intestinal epithelial cells. These transporters, including many other urate transporters, interact with PDZK1 through specific C-terminal binding sequences (Table 1). PDZK1 has a strong genetic association with sUA levels, suggesting that PDZK1 functions as a scaffolding protein to assemble the various transporters into a "uric acid transportasome" for efficient control of urate transport and homeostasis (Figure 3) [20].

The urate transport activity of URAT1 depends on a system of other transporters (yellow in Figure 3). The primary active transporter $\mathrm{Na}^{+} / \mathrm{K}^{+}$ATPase provides an inward sodium ion gradient (higher concentration of extracellular sodium ions). The secondary active transporters, the sodium monocarboxylate cotransporters SMCT1/SLC5A8 and SMCT2/SLC5A12, use the sodium ion gradient for the inward cotransport of lactate and other monocarboxylates. Finally, URAT1 is the tertiary active transporter in this system, requiring the counterion lactate/monocarboxylate gradient generated by SMCT1/2 for the inward transport of urate. Furthermore, we suggest that the sodium/proton exchanger NHE3/SLC9A3, which imports sodium ions and exports protons, may also be important for URAT1 activity. This system of transporters that is indirectly involved in import of urate from the lumen of the proximal tubule are 
sensitive to certain drugs that elevate sUA levels by increasing the urate transport activity of URAT1. For example, diuretics reduce the urinary excretion of urate and elevate sUA levels, possibly through enhanced sodium reabsorption in the proximal tubule. This effect would elevate the intracellular levels of lactate within the renal proximal epithelial cells (through SLC5A8/12) and also lower the extracellular pH (through NHE3), enhancing the reabsorption of urate by URAT1.

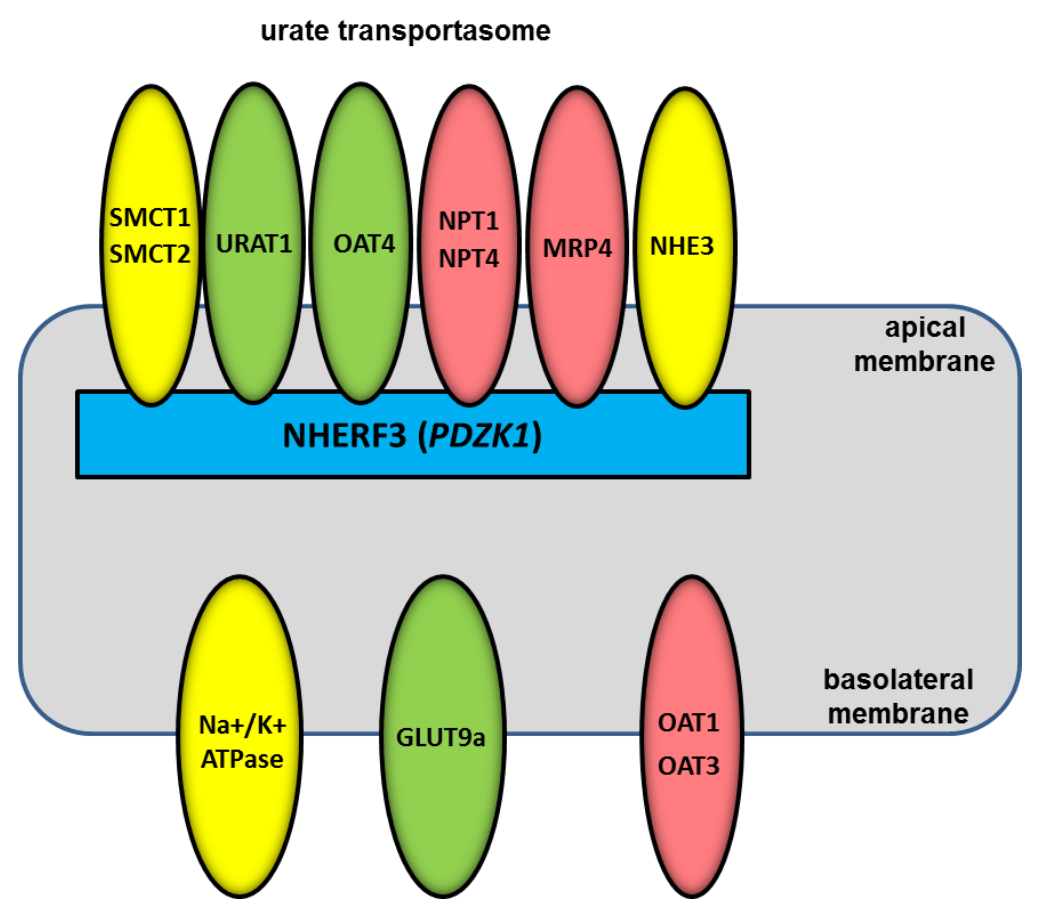

Figure 3. The scaffolding protein NHERF3/PDZK1 clusters apical membrane urate transporters into a "urate transportasome" for more efficient urate handling in the kidney. Transporters indicated in yellow do not transport urate, but are important for the transport of urate by URAT1 (see text for further details). For simplicity, certain transporters are grouped together (e.g. NPT1 and NPT4).

\section{GLUT9}

The SLC family 2A, facilitated glucose transporter member 9 (GLUT9/SLC2A9), like URAT1, is also essential for the efficient reabsorption of urate from the renal proximal tubule (Figure 1a). Individuals carrying inactivating mutations in GLUT9 have renal hypouricemia and very high levels of urinary uric acid excretion. In some individuals with inactivating GLUT9 mutations, the fractional excretion of uric acid exceeds $100 \%$ (meaning that the amount of renally excreted uric acid exceeds the amount of glomerularfiltered uric acid), providing evidence that uric acid is actively secreted in the kidneys (red arrow in Figure 1). The long isoform of GLUT9 (GLUT9a or -L) is localized to the basolateral membrane of RPTECs, and transports uric acid along its concentration gradient from inside the cells to the blood, completing the process of uric acid reabsorption that is initiated by URAT1 (Figure 1a). Unlike URAT1, there are no medications that target GLUT9. Similar to other members of the SLC2 family, GLUT9 has been shown to transport glucose and other sugars, although this has recently been questioned [21]. However, sugars may influence the transport of urate by GLUT9 [22], suggesting that dietary sugars can influence sUA levels through GLUT9. A short isoform of GLUT9 (GLUT9b or -S) differs at the amino terminus from the longer isoform due to alternative splicing. An antibody raised against the unique 21 amino terminal amino acids of GLUT9b detected this variant on membranes of epithelial cells in the collecting duct [23], suggesting the possibility that this distal segment of the nephron handles urate. Interestingly, this antibody did not detect GLUT9b on the proximal tubule. 


\section{OAT4}

OAT4/SLC22A11 is expressed on the apical membrane of RPTECs (Figure 1a), has a $51 \%$ amino acid identity to URAT1, and has a strong genetic association with sUA levels. However, OAT4 is a weak transporter of uric acid in vitro, and its role in vivo in uric acid transport and homeostasis is unclear. Inactivating mutations of OAT4 have been reported [24] but the sUA levels in the individuals carrying these mutations is unknown. Similar to URAT1, OAT4 is an organic anion exchanger, and diuretics such as hydrochlorothiazide were shown to enhance urate import by OAT4 (trans-stimulation), explaining a possible OAT4-dependent mechanism for diuretic-induced hyperuricemia [25]. However, to our knowledge these studies have not yet been replicated, and mixed findings have been reported on the genetic association of OAT4 with diuretic-associated gout $[26,27]$. OAT4 is not expressed in mice, and no specific OAT4 inhibitors are known (lesinurad and probenecid inhibit OAT4, but also other transporters), hampering efforts to study OAT4 using genetic or pharmacological methods.

\section{OAT10}

OAT10/SLC22A13 was initially characterized as a urate transporter that is expressed on apical membranes of proximal tubule cells and trans-stimulated by cyclosporine, explaining a mechanism for cyclosporine-induced hyperuricemia [28]. This localization is supported by a subsequent report using different antibodies [29], but is inconsistent with a third study which shows localization on basolateral membranes of the collecting duct [30]. OAT10, however, has a consensus C-terminal PDZ recognition motif (Table 1) for localization to apical membranes of proximal tubule cells. No genetic association of OAT10 with sUA levels has been found, and the role of OAT10 in urate transport and homeostasis needs to be further characterized.

Table 1. Carboxyl (C-) terminal sequences of transporters known or presumed to interact with PDZK1.

\begin{tabular}{|l|l|l|l|}
\hline Location & Transporter & C-terminal Sequence & Reference \\
\hline URAT1/SLC22A12 & STQF & Anzai 2004 [31] \\
\hline OAT4/SLC22A11 & STSL & Miyazaki 2005 [32] \\
\hline OAT10/SLC22A13 & STYF & Bahn 2008 [28 $]^{\text {a }}$ \\
\hline SMCT1/SLC5A8 & GTRL & Kanai 2008 [33] \\
\hline Kidney & SMCT2/SLC5A12 & TTHF & Kanai 2008 [33] \\
\hline NPT1/SLC17A1 & HTRL & Gisler 2003 [34] \\
\hline NPT4/SLC17A3 & LTRL & Jutabha 2010 [35] \\
\hline MRP2/ABCC2 & STKF & Kocher 1999 [36] \\
\hline MRP4/ABCC4 & ETAL & Park 2014 [37] \\
\hline NHE3/SLC9A3 & STHM & Gisler 2003 [34] \\
\hline Intestine & NPT5/SLC17A4 & FTHL & Togawa 2012 [38] \\
\hline
\end{tabular}

*BCRP may interact with PDZK1 in a non-classical way via an internal sequence.

${ }^{a, b}$ Apical membrane localization in RPTEC or enterocytes $^{\mathrm{b}}$. Presumed to interact with PDZK1 based on C-terminal sequence. 


\section{OAT1 and OAT3}

OAT1/SLC22A6 and OAT3/SLC22A8 are important transporters involved in the renal secretion of many drugs. They are localized to the basolateral membranes of RPTECs and transport urate in vitro, suggesting that they are involved in urate secretion in vivo [40]. However, OAT1 and OAT3 show no genetic association with sUA levels, and there is no solid evidence supporting a role in uric acid homeostasis in humans. OAT1 and OAT3 are in the same transporter family and have a $46 \%$ and $42 \%$ amino acid identity with URAT1 [41]. The URAT1 inhibitor probenecid also inhibits OAT1 and OAT3, leading to OAT1/3dependent drug-drug interactions that limits its use. The URAT1 inhibitor lesinurad has detectable interactions with OAT1 and OAT3 in vitro, but in contrast to probenecid has no clinically relevant OAT1/3dependent drug-drug interactions $[42,43]$. Because of the relatedness to URAT1, all URAT1 inhibitors under evaluation for treatment of hyperuricemia associated with gout should be evaluated against OAT1 and OAT3.

\section{NPT1, NPT4 and NPT5}

NPT1/SLC17A1, NPT4/SLC17A3, and NPT5/SLC17A4 (formerly known as NPT-like) occur in a gene cluster with a strong genetic association with sUA levels [7]. NPT1 and NPT4 localize to the apical membrane of RPTECs and are hypothesized to export urate into the tubule lumen as part of the urate secretory pathway. Support for this functional role comes from the identification and analysis of missense mutations involved in hyperuricemia and/or gout that exhibit altered urate transport activities [35, 44-47]. Moreover, it has been suggested that hyperuricemia induced by diuretics may be due to inhibition of NPT4 [45]. Meanwhile, NPT5 is a urate exporter that expressed on the apical surface of intestinal epithelial cells, suggesting a role in intestinal urate secretion [38].

\section{MRP4}

Multi-resistance protein 4 (MRP4/ABCC4) transports urate in vitro, localizes to the apical membranes of RPTEC, and was recently shown to be genetically associated with gout and renal excretion of uric acid [8]. Consistent with its proposed role as an efflux transporter involved in the renal secretion of urate, a nonsynonymous variant P1036L of MRP4 had reduced uric acid transport activity [6]. Diuretics have been proposed to elevate sUA levels through inhibition of MRP4 [48].

\section{$A B C G 2$}

ATP-binding cassette sub-family G member 2 ( $A B C G 2$, also known as breast cancer-resistance protein or $B C R P$, encoded by the $A B C G 2$ gene) is an ATP-dependent transporter that is expressed on the apical membranes of RPTEC and intestinal epithelia, involved in the export of urate (secretion), and with a strong genetic association with sUA levels and gout. Humans carrying a common genetic variant, Q141K, which exhibits reduced expression and activity, have elevated SUA levels and elevated renal excretion of uric acid, supporting a role for ABCG2 in the intestinal secretion of uric acid [49]. ABCG2 is strongly expressed in the intestine and is weakly expressed in the kidneys, suggesting that it primarily functions to transport urate into the intestinal lumen. The strong genetic association with sUA and gout suggests that drugs that activate $A B C G 2$ could serve as gout therapies.

\section{Evolution of gout in humans}

Uric acid is a small, seemingly innocuous waste compound found mainly in the excreta from many different species. Biochemically, this simple compound is merely one step in the removal pathway for 
nitrogenous wastes in reptiles and birds and for purines in humans. The degradation of purines from nucleic acid results in the production of uric acid, which in most mammals is instantly converted to allantoin by the enzyme uricase. However, about 43 million years ago, for unknown but likely interesting reasons, selective pressure mounted for higher blood levels of uric acid. Primates carrying mutations in the uric acid handling pathways that resulted in higher uric acid levels experienced a selective advantage. This pressure continued for 20 million years during simian evolution steadily decreasing uricase activity and increasing renal uric acid transporter affinity for uric acid, resulting in humans with no uricase enzyme activity, a high affinity renal uric acid reabsorbing transporter and the highest uric acid levels of any animal in the class Mammalia [50]. The hypotheses explaining the rise in sUA are many [3], and may involve enhanced energy storage in resource-constrained environments due to biochemical effects of uric acid on metabolism. These same selective pressures no longer exist today and humans are left with a uric acid load that in a time of resource excess has created an epidemic of gout and potentially other comorbidities associated with uric acid [51]. Despite the strength of the association between uric acid and cardiovascular disease, hypertension and chronic kidney disease, these studies do not provide causation, thus it is unclear if uric acid levels are the culprit in driving these illnesses. More recent studies utilizing mendelian randomization and interventional urate lowering drugs have suggested that some aspects of the comorbidities are not caused by uric acid $[52,53]$ and others indicate that uric acid is causing these comorbidities [54,55]. The data for these findings is actively being developed thus the overall picture will certainly change in the coming years. This is important because if uric acid is participating in these comorbidities, then urate lowering therapy could well be an important additional treatment for vascular disease, which could work in addition to current therapies.

In humans, uric acid is produced by the degradation of endogenous purines and the purines in food. There are three primary sources of uric acid in the body: 1) metabolism, whereby free circulating purines are degraded in the liver and intestines by xanthine oxidase into uric acid, 2) ongoing cellular degradation (releasing DNA and RNA), which are degraded into uric acid, and 3) diet has a smaller impact through the ingestion of purines and nucleic acids and subsequent metabolism of these compounds in the intestinal mucosa by resident xanthine oxidase.

The effects of dietary purines on serum uric acid levels is relatively mild with even strict diets affecting uric acid levels marginally [56]. Purine increases from resulting from fructose, beer (from yeast) and other purine rich foods is of primary concern. Fructose ingestion depletes ATP from the action of fructokinase. The depleted ATP (AMP) is converted to uric acid through the purine catabolic pathway.

Once produced, urate cannot be degraded by the human body so all urate must be eliminated either by the kidney or the intestine. Two-thirds of urate elimination is through the kidneys, the remainder through the intestines. The balance of production and elimination determines the concentration of uric acid in the serum as described above.

\section{Genetics of uric acid}

Genome-wide association studies (GWAS) have identified 28 separate loci that control hyperuricemia and gout. Many of these code for transporters directly involved in the regulation of uric acid levels in humans. These include transporters found in the kidney, liver and intestine with functions spanning both uric acid secretion and resorption [57].

GWAS studies also show influence of urate production (glucokinase regulator or GCKR, inhibin-activin network, insulin) - links to glucose homeostasis, diabetes, feeding status, energy expenditure. 
Interestingly, xanthine oxidase, and other enzymes in the uric acid biosynthetic pathway are not found in GWAS studies suggesting that despite a clear role, apparently genetically available variations in the activity of these enzymes does not lead to alterations in uric acid levels. The work in this area is proceeding rapidly with ongoing resequencing projects and expression analysis in clinical samples.

\section{Renal handling of uric acid}

In early studies on renal apical BBMVs (brush-border membrane vesicles) and basolateral vesicles, transport activities were characterized [58] and eventually led to the cloning of uric acid transporters from renal tissue and the discovery of a multiprotein transportasome functioning to regulate uric acid levels at the apical membrane $[59,60]$.

In a normal human kidney, clearance of urate is a function of glomerular filtration rate (GFR) and fractional excretion of uric acid. Urate is freely filtered at the glomerulus and is subsequently almost entirely reabsorbed within the proximal tubule. Secretion of urate also occurs, either concomitantly with reabsorption or subsequent to reabsorption. The rate of urate excretion as a percentage of the rate of filtration of urate is termed the fractional excretion of uric acid (FEUA).

Low FEUA correlates with increased sUA and the characterization of the efficiency of urate secretion is typically done by measuring FEUA. Gout patients often exhibit significantly decreased FEUA. In addition, decreased GFR also increases SUA, so patients with low FEUA, or impaired renal function are in danger of hyperuricemia and gout

\section{Renal uric acid reabsorption}

Most uric acid that is filtered by the glomerulus is reabsorbed in the proximal tubule. The main transporter on the apical side of the tubule is URAT1, however OAT4 and OAT10 are also possible contributors to uric acid resorption across the apical membrane. It is likely that the sole transporter on the basolateral side is GLUT9. This is because the phenotype of GLUT9 mutations in humans results in an FEUA at or above $100 \%$, suggesting complete loss of resorption [61]. In contrast, the effect of URAT1 mutations on FEUA is less severe $>50 \%$ [62]. Other transporters may be present on the apical side but it is believed that only GLUT9 functions to export urate to the interstitium (blood side) across the basolateral membrane.

The sensitivity of the kidney to alterations in transporter function is also detected at the level of gene expression of the transporter URAT1. In women, URAT1 expression is likely down regulated by estrogens resulting in fewer transporters at the apical surface and GFR is increased by estrogen through renal vasodilation.

In mice, URAT1 and GLUT9 expression is decreased in the presence of estradiol consistent with premenopausal women having significantly lower uric acid levels that post-menopausal women [63, 64]. Further confirmation of this effect is obtained through testing of uric acid levels in patients undergoing gender reassignment. Addition of estrogen lowers uric acid levels and addition of androgen raises them in these patients [65].

\section{Reabsorption transporters}

\section{Cyclical uric acid transport at the proximal tubule membrane}

The result of import and export transporters coexpressed and functioning on both the apical and 
basolateral sides of the tubular membrane means that uric acid likely cycles through the kidney multiple times during the day and perhaps within the same tubule. The renal system filters approximately 180 liters of water each day, similarly for uric acid each molecule of uric acid is likely subjected to multiple rounds of filtration, resorption and /or secretion. The relative affinity and capacity curves for these transporters govern the percentage of uric acid that is resorbed, secreted and the percentage that finally ends up excreted in urine [41]. A similar process is likely also occurring in the intestine.

URAT1 inhibitors used for the treatment of gout (Figure 4)

\section{Benzbromarone, (3,5-dibromo-4-hydroxyphenyl)-(2-ethyl-1-benzofuran-3-yl)methanone}

Benzbromarone was originally derived from benziodarone, an iodinated compound synthesized initially for coronary dilation. Benziodarone was withdrawn from the market in Europe due to instances of jaundice in patients treated with this compound. Prior to the withdrawal, it was noted that benziodarone was an effective uric acid lowering agent. The brominated analog of benziodarone was synthesized as a follow up for uric acid lowering in patients with gout (benzbromarone) [66]. This compound was withdrawn from the market later due to idiosyncratic liver toxicity and is only used in Europe on a named patient basis.

Benzbromarone is capable of blocking URAT1 potently and selectively, it is relatively weak on both OAT4 and on GLUT9. The pharmacokinetics of benzbromarone provide for a $C_{\max }$ of approximately $5 \mu \mathrm{M}$ [67]. This is well within the potency on URAT1 at $100 \mathrm{nM}$, however, the high $\mu \mathrm{M}$ potency on other transporters (GLUT9 AND OAT4) in vitro, coupled with the relatively poor renal excretion profile suggests that benzbromarone will primarily inhibit URAT1 to produce its uricosuric effect.
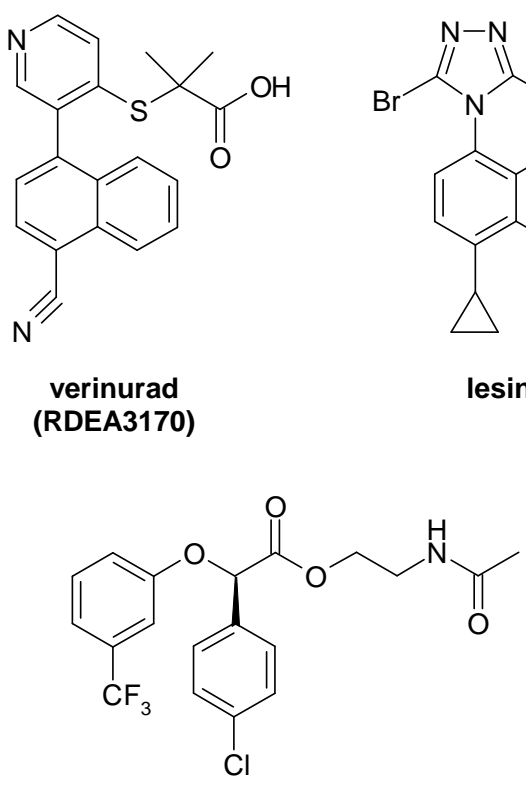

arhalofenate

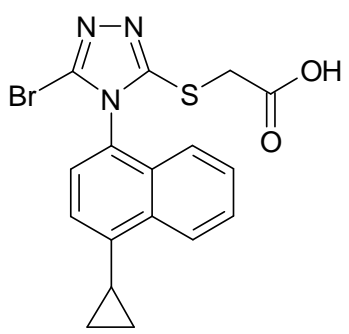

lesinurad

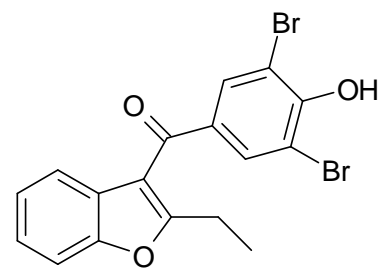

benzbromarone

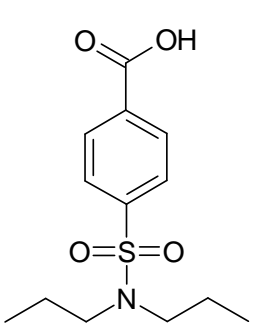

probenecid

Figure 4. Structure of URAT1 inhibitors for the treatment of gout. Verinurad and arhalofenate are currently under evaluation in clinical trials.

\section{Probenecid, 4-[(dipropylamino) sulfony1] benzoic acid}

Probenecid is a compound originally developed by Bayer to reduce the renal excretion of penicillin given the difficulty in obtaining sufficient quantities of antibiotic to cover the overwhelming need at that time. Probenecid is an inhibitor of OAT1 and OAT3, transporters responsible for renal excretion of many drugs not just antibiotics, thus use of probenecid is limited due to the significant drug-drug interactions. 
After the use of probenecid became widespread in combination with antibiotics, it was discovered that it also reduced serum uric acid levels by inhibition of renal reabsorption, thus making it useful for the treatment of gout. The compound is still used for this purpose today, though the drug - drug interactions, multiple daily dosing and generally weak efficacy limit its use overall [68].

\section{Lesinurad (RDEA594), 2-((5-bromo-4-(4-cyclopropylnaphthalen-1-yl)-4H-1,2,4-triazol-3-yl)thio)acetic acid}

Lesinurad was discovered as an active metabolite from an unrelated clinical program. Patients given the parent compound experienced a profound uric acid lowering effect. This effect was due to drug-mediated alterations in fractional excretion of uric acid [43]. This result suggested renal transporter inhibition as the mechanism. One metabolite from the parent was preferentially excreted to urine (RDEA594). Purification and testing for URAT1 inhibition provided evidence that RDEA594 (lesinurad) was responsible and likely mediated its effects through inhibition of URAT1. This was later confirmed with human PK / PD studies in phase 1 [43].

Lesinurad is a molecule capable of significant uric acid lowering in the presence of an XO inhibitor via the inhibition of URAT1. This inhibition increases the FEUA in patients taking this medicine which targets the defect found in most gout patients. Combination therapy showed a beneficial profile compared to monotherapy allopurinol, however significant numbers of patients experienced creatinine elevations while on lesinurad thus careful renal monitoring by prescribing physicians is required.

\section{Verinurad (RDEA3170), 2-((3-(4-cyanonaphthalen-1-yl)pyridin-4-yl)thio)-2-methylpropanoic acid}

Verinurad is a selective uric acid reabsorption inhibitor with nM potency against URAT1 [69]. It is in clinical development for the treatment of gout and hyperuricemia in combination with a xanthine oxidase inhibitor. In constrast to lesinurad, in phase 2 studies in combination with a xanthine oxidase inhibitor, verinurad treatment has not resulted in serum creatinine elevations, likely due to its pharmacodynamic/pharmacokinetic profile and the action of the xanthine oxidase inhibitor reducing the urinary uric acid concentration and thereby reducing the incidence of uric acid crystallization and $\mathrm{sCr}$ elevations. The combination of verinurad and a xanthine oxidase inhibitor provided greater reductions in sUA than by a xanthine oxidase inhibitor alone even after doubling the dose of the XOI [70].

\section{Arhalofenate, 2-acetamidoethyl (2R)-2-(4-chlorophenyl)-2-[3-(trifluoromethyl)phenoxy]acetate}

This URAT1 inhibitor is currently in clinical trials for gout [71]. Arhalofenate when dosed at $800 \mathrm{mg}$ per day reduced serum uric acid similarly to $300 \mathrm{mg}$ of allopurinol. Interestingly, this treatment also decreased gout flares significantly compared to allopurinol at a dosage of $300 \mathrm{mg}$. Arhalofenate was well tolerated in early trials. In addition to its uricosuric properties, it also appears to be capable of reducing inflammation. This effect could lead to reductions in flares during treatment though the data to date is inconclusive. Combination studies are underway and development is continuing in the US.

\section{Binding of known inhibitors}

The interactions between uricosuric agents and URAT1 has been studied using modeling and chimeric mapping studies [41]. This work has demonstrated that the URAT1 inhibitors (lesinurad, probenecid, benzbromarone and RDEA3170 (verinurad) all bind to the same site in the transporter [41]. There are interesting differences in the specific amino acids required for binding for each compound but this is not surprising given the structural differences between them. It's likely that there is a specific characteristic of this location within the transporter that makes it susceptible to inhibition by a wide variety of compounds. 
One of these characteristics may be that uric acid itself appears to interact with this pocket. Modeling of the relevant amino acids onto and OAT1 scaffold suggests that these amino acids are located within the interior channel of the transporter, ideally positioned to bind either substrate or inhibitor [41].

\section{Conclusions}

Human uric acid level in serum is regulated by a complex array of multi-protein complexes found in several different organs. Evolutionary pressure to increase uric acid levels likely resulted in the need to regulate these levels. The kidney plays the key role by sensing current uric acid levels from the blood in the filtrate and adjusting reabsorption accordingly. The intestine is also likely capable of sensing and responding to serum uric acid levels, however, it is less well characterized. The risks of elevated uric acid are well characterized and a number of therapies have been developed to reduce these levels in patients with gout. URAT1 inhibitors represent one of the two main approaches for reduction of serum uric acid and work by increasing the efficiency of uric acid excretion. The other relies on the blockade production of uric acid via inhibition of xanthine oxidase. Combination therapy with both an excretion enhancer and a production inhibitor is a powerful approach to efficient urate lowering therapy.

\section{References}

[1] D. Khanna, J.D. Fitzgerald, P.P. Khanna, S. Bae, M.K. Singh, T. Neogi, et al. Arthritis care \& research 64 (2012) 1431-1446.

[2] S.G. Mallat, S. Al Kattar, B.Y. Tanios, A. Jurjus, Current hypertension reports 18 (2016) 74.

[3] J.T. Kratzer, M.A. Lanaspa, M.N. Murphy, C. Cicerchi, C.L. Graves, P.A. Tipton, E.A. et al. Proceedings of the National Academy of Sciences of the United States of America 111 (2014) 3763-3768.

[4] M.B. Bridgeman, B. Chavez, Expert opinion on pharmacotherapy 16 (2015) 395-398.

[5] K. Sakhaee, Journal of nephrology 27 (2014) 241-245.

[6] M. Kolz, T. Johnson, S. Sanna, A. Teumer, V. Vitart, M. Perola, et al. PLoS genetics 5 (2009) e1000504.

[7] A. Kottgen, E. Albrecht, A. Teumer, V. Vitart, J. Krumsiek, C. Hundertmark, et al. Nature genetics 45 (2013) 145-154.

[8] C. Tanner, J. Boocock, E.A. Stahl, A. Dobbyn, A.K. Mandal, M. Cadzow, A.J. Phipps-Green, R.K. Topless, J. Harre Hindmarsh, L.K. Stamp, N. Dalbeth, H.K. Choi, D.B. Mount, T.R. Merriman, Arthritis \& rheumatology (2017).

[9] X. Xu, C. Li, P. Zhou, T. Jiang, Pharmaceutical biology 54 (2016) 3151-3155.

[10] T. Takada, K. Ichida, H. Matsuo, A. Nakayama, K. Murakami, Y. Yamanashi, H. Kasuga, N. Shinomiya, H. Suzuki, Nucleosides, nucleotides \& nucleic acids 33 (2014) 275-281.

[11] A. Nakayama, H. Matsuo, T. Takada, K. Ichida, T. Nakamura, Y. Ikebuchi, K. Ito, T. Hosoya, Y. Kanai, H. Suzuki, N. Shinomiya, Nucleosides, nucleotides \& nucleic acids 30 (2011) 1091-1097.

[12] A. Hosomi, T. Nakanishi, T. Fujita, I. Tamai, PloS one 7 (2012) e30456.

[13] M. Hiratochi, K. Tatani, K. Shimizu, Y. Kuramochi, N. Kikuchi, N. Kamada, F. Itoh, M. Isaji, European journal of pharmacology 690 (2012) 183-191.

[14] S. Liu, F. Perez-Ruiz, J.N. Miner, Joint, bone, spine : revue du rhumatisme 84 (2017) 183-188.

[15] A. Enomoto, H. Kimura, A. Chairoungdua, Y. Shigeta, P. Jutabha, S.H. Cha, et al. Nature 417 (2002) 447-452.

[16] P.H. Gois, E.R. Souza, The Cochrane database of systematic reviews (2013) CD008652.

[17] A.S. Kydd, R. Seth, R. Buchbinder, L. Falzon, C.J. Edwards, D.M. van der Heijde, C. Bombardier, The Journal of rheumatology. Supplement 92 (2014) 33-41. 
[18] C.S. Lieber, D.P. Jones, M.S. Losowsky, C.S. Davidson, The Journal of clinical investigation 41 (1962) 1863-1870.

[19] J. Padova, G. Bendersky, The New England journal of medicine 267 (1962) 530-534.

[20] R.L. George, R.T. Keenan, Current rheumatology reports 15 (2013) 309.

[21] K. Ebert, M. Ludwig, K.E. Geillinger, G.C. Schoberth, J. Essenwanger, J. Stolz, H. Daniel, H. Witt, The Journal of membrane biology 250 (2017) 171-182.

[22] K. Witkowska, K.M. Smith, S.Y. Yao, A.M. Ng, D. O'Neill, E. Karpinski, J.D. Young, C.I. Cheeseman, American journal of physiology. Renal physiology 303 (2012) F527-539.

[23] T. Kimura, M. Takahashi, K. Yan, H. Sakurai, PloS one 9 (2014) e84996.

[24] J.E. Shima, T. Komori, T.R. Taylor, D. Stryke, M. Kawamoto, S.J. Johns, E.J. Carlson, T.E. Ferrin, K.M. Giacomini, American journal of physiology. Renal physiology 299 (2010) F767-775.

[25] Y. Hagos, D. Stein, B. Ugele, G. Burckhardt, A. Bahn, Journal of the American Society of Nephrology: JASN 18 (2007) 430-439.

[26] M.A. McAdams-DeMarco, J.W. Maynard, A.N. Baer, L.W. Kao, A. Kottgen, J. Coresh, Annals of the rheumatic diseases 72 (2013) 701-706.

[27] Y. Bao, G. Curhan, T. Merriman, R. Plenge, P. Kraft, H.K. Choi, Annals of the rheumatic diseases 74 (2015) 1394-1398.

[28] A. Bahn, Y. Hagos, S. Reuter, D. Balen, H. Brzica, W. Krick, B.C. Burckhardt, I. Sabolic, G. Burckhardt, The Journal of biological chemistry 283 (2008) 16332-16341.

[29] L. Fagerberg, B.M. Hallstrom, P. Oksvold, C. Kampf, D. Djureinovic, J. Odeberg, et al. Molecular \& cellular proteomics : MCP 13 (2014) 397-406.

[30] C. Schulz, C. Fork, T. Bauer, S. Golz, A. Geerts, E. Schomig, D. Grundemann, The Biochemical journal 457 (2014) 243-251.

[31] N. Anzai, H. Miyazaki, H. Endou, Seikagaku. The Journal of Japanese Biochemical Society 76 (2004) 101-110.

[32] H. Miyazaki, N. Anzai, S. Ekaratanawong, T. Sakata, H.J. Shin, P. Jutabha, T. Hirata, X. He, H. Nonoguchi, K. Tomita, Y. Kanai, H. Endou, Journal of the American Society of Nephrology : JASN 16 (2005) 3498-3506.

[33] Y. Kanai, Nihon rinsho. Japanese journal of clinical medicine 66 (2008) 659-666.

[34] S.M. Gisler, S. Pribanic, D. Bacic, P. Forrer, A. Gantenbein, L.A. Sabourin, A. Tsuji, Z.S. Zhao, E. Manser, J. Biber, H. Murer, Kidney international 64 (2003) 1733-1745.

[35] P. Jutabha, N. Anzai, K. Hayashi, M. Domae, K. Uchida, H. Endou, H. Sakurai, Journal of pharmacological sciences 116 (2011) 392-396.

[36] O. Kocher, N. Comella, A. Gilchrist, R. Pal, K. Tognazzi, L.F. Brown, J.H. Knoll, Laboratory investigation; a journal of technical methods and pathology 79 (1999) 1161-1170.

[37] J. Park, J.O. Kwak, B. Riederer, U. Seidler, S.P. Cole, H.J. Lee, M.G. Lee, Journal of the American Society of Nephrology: JASN 25 (2014) 726-736.

[38] N. Togawa, T. Miyaji, S. Izawa, H. Omote, Y. Moriyama, American journal of physiology. Cell physiology 302 (2012) C1652-1660.

[39] T. Shimizu, T. Sugiura, T. Wakayama, A. Kijima, N. Nakamichi, S. Iseki, D.L. Silver, Y. Kato, Drug metabolism and disposition: the biological fate of chemicals 39 (2011) 2148-2154.

[40] S.K. Nigam, K.T. Bush, G. Martovetsky, S.Y. Ahn, H.C. Liu, E. Richard, V. Bhatnagar, W. Wu, Physiological reviews 95 (2015) 83-123.

[41] P.K. Tan, T.M. Ostertag, J.N. Miner, Scientific reports 6 (2016) 34995.

[42] J. Miner, P.K. Tan, D. Hyndman, S. Liu, C. Iverson, P. Nanavati, D.T. Hagerty, K. Manhard, Z. Shen, J.L. Girardet, L.T. Yeh, R. Terkeltaub, B. Quart, Arthritis research \& therapy 18 (2016) 214.

[43] Z. Shen, C. Rowlings, B. Kerr, V. Hingorani, K. Manhard, B. Quart, L.T. Yeh, C. Storgard, Drug design, development and therapy 9 (2015) 3423-3434. 
[44] P. Jutabha, N. Anzai, T. Kimura, A. Taniguchi, W. Urano, H. Yamanaka, H. Endou, H. Sakurai, Journal of pharmacological sciences 115 (2011) 249-253.

[45] P. Jutabha, N. Anzai, M.F. Wempe, S. Wakui, H. Endou, H. Sakurai, Nucleosides, nucleotides \& nucleic acids 30 (2011) 1302-1311.

[46] T. Chiba, H. Matsuo, Y. Kawamura, S. Nagamori, T. Nishiyama, L. Wei, et al. Arthritis \& rheumatology 67 (2015) 281-287.

[47] M. Sakiyama, H. Matsuo, S. Nagamori, W. Ling, Y. Kawamura, A. Nakayama, T. Higashino, T. Chiba, K. Ichida, Y. Kanai, N. Shinomiya, Nucleosides, nucleotides \& nucleic acids 35 (2016) 536-542.

[48] A.A. El-Sheikh, J.J. van den Heuvel, J.B. Koenderink, F.G. Russel, British journal of pharmacology 155 (2008) 1066-1075.

[49] N. Dalbeth, M.E. House, G.D. Gamble, B. Pool, A. Horne, L. Purvis, A. Stewart, M. Merriman, M. Cadzow, A. Phipps-Green, T.R. Merriman, Arthritis research \& therapy 16 (2014) R34.

[50] P.K. Tan, J.E. Farrar, E.A. Gaucher, J.N. Miner, Molecular biology and evolution 33 (2016) $2193-$ 2200.

[51] R.J. Johnson, Y.Y. Sautin, W.J. Oliver, C. Roncal, W. Mu, L. Gabriela Sanchez-Lozada, B. RodriguezIturbe, T. Nakagawa, S.A. Benner, J Comp Physiol B 179 (2009) 67-76.

[52] A.J. Ahola, N. Sandholm, C. Forsblom, V. Harjutsalo, E. Dahlstrom, P.H. Groop, G. FinnDiane Study, Kidney international (2017).

[53] I. Sluijs, M.V. Holmes, Y.T. van der Schouw, J.W. Beulens, F.W. Asselbergs, J.M. Huerta, et al. Diabetes 64 (2015) 3028-3036.

[54] D. Yan, J. Wang, F. Jiang, R. Zhang, T. Wang, S. Wang, D. Peng, Z. He, H. Chen, Y. Bao, C. Hu, W. Jia, International journal of cardiology 214 (2016) 194-199.

[55] J. White, R. Sofat, G. Hemani, T. Shah, J. Engmann, C. Dale, et al. The lancet. Diabetes \& endocrinology 4 (2016) 327-336.

[56] T. Yamamoto, H. Yokoyama, Y. Moriwaki, S. Takahashi, M. Suda, T. Hada, K. Higashino, European journal of clinical nutrition 44 (1990) 659-664.

[57] T.R. Merriman, Arthritis research \& therapy 17 (2015) 98.

[58] T. Dan, H. Koga, European journal of pharmacology 187 (1990) 303-312.

[59] E. Leal-Pinto, W. Tao, J. Rappaport, M. Richardson, B.A. Knorr, R.G. Abramson, The Journal of biological chemistry 272 (1997) 617-625.

[60] N. Anzai, Y. Kanai, H. Endou, Current opinion in rheumatology 19 (2007) 151-157.

[61] D. Dinour, N.K. Gray, S. Campbell, X. Shu, L. Sawyer, W. Richardson, et al. Journal of the American Society of Nephrology : JASN 21 (2010) 64-72.

[62] A. Ochi, T. Takei, A. Ichikawa, C. Kojima, T. Moriyama, M. Itabashi, T. Mochizuki, A. Taniguchi, K. Nitta, Clinical and experimental nephrology 16 (2012) 316-319.

[63] Y. Takiue, M. Hosoyamada, M. Kimura, H. Saito, Nucleosides, nucleotides \& nucleic acids 30 (2011) 113-119.

[64] F.M. Anton, J. Garcia Puig, T. Ramos, P. Gonzalez, J. Ordas, Metabolism: clinical and experimental 35 (1986) 343-348.

[65] E. Lioudaki, E.S. Ganotakis, D.P. Mikhailidis, D.R. Nair, Current pharmaceutical design 16 (2010) 3815-3822.

[66] C.G. Wermuth, The practice of medicinal chemistry, 2nd ed., Academic, Amsterdam ; London, 2003.

[67] I. Walter-Sack, J.X. de Vries, A. von Bubnoff, V. Pfleilschifter, R. Raedsch, European journal of medical research 1 (1995) 16-20.

[68] N. Robbins, S.E. Koch, M. Tranter, J. Rubinstein, Cardiovascular toxicology 12 (2012) 1-9.

[69] P.K. Tan, S. Liu, E. Gunic, J.N. Miner, Scientific reports 7 (2017) 665. 
[70] C. Diaz-Torne, N. Perez-Herrero, F. Perez-Ruiz, Current opinion in rheumatology 27 (2015) 164-169.

[71] J. Poiley, A.S. Steinberg, Y.J. Choi, C.S. Davis, R.L. Martin, C.A. McWherter, P.F. Boudes, I. Arhalofenate Flare Study, Arthritis \& rheumatology 68 (2016) 2027-2034.

(C)2017 by the authors; licensee IAPC, Zagreb, Croatia. This article is an open-access article distributed under the terms and conditions of the Creative Commons Attribution license (http://creativecommons.org/licenses/by/3.0/) (cc)) EY 


\section{Supplementary figure}

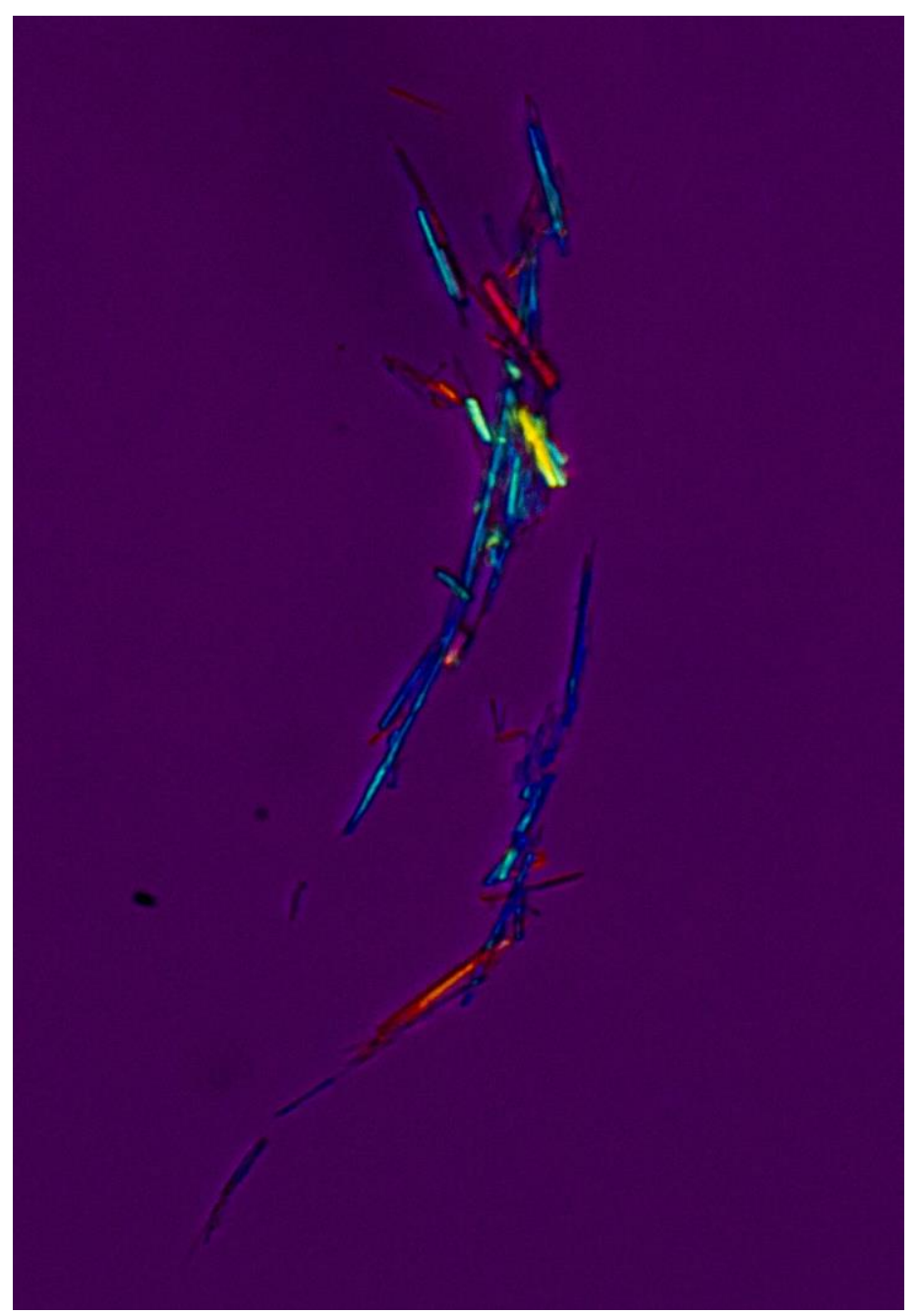

Figure SP1. Photomicrograph under polarized light of negatively birefringent monosodium uric acid crystals, the underlying cause of gouty inflammation 